\title{
GAIA Antenatal Bleeding Level of Diagnostic Certainty
}

National Cancer Institute

\section{Source}

National Cancer Institute. GAIA Antenatal Bleeding Level of Diagnostic Certainty. NCI

Thesaurus. Code C128670.

A classification of maternal and fetal outcomes relating to antenatal bleeding, developed by the Global Alignment of Immunization safety Assessment in pregnancy, based on the extent to which the diagnosis has been confirmed. 